\title{
Macrofauna del suelo y condiciones edafoclimáticas en un gradiente altitudinal de zonas cafeteras, Huila, Colombia
}

\author{
Leidy Machado Cuellar ${ }^{1}$, Leonardo Rodríguez Suárez ${ }^{1 *}$, Valentín Murcia Torrejano ${ }^{1}$, \\ Sergio Andrés Orduz Tovar ${ }^{1}$, Claudia Mercedes Ordoñez Espinosa ${ }^{1} \&$ Juan Carlos Suárez ${ }^{2}$ \\ 1. Centro de Formación Agroindustrial La Angostura, SENNOVA - Grupo de Investigación Agroindustrial La Angostura \\ - Servicio Nacional de Aprendizaje - SENA, Campoalegre - Huila, Colombia; leidymachadocuellar@gmail.com, \\ 1.rodriguezsua@gmail.com,vmurcia4@misena.edu.co, saorduz6@misena.edu.co,clmechas24@misena.edu.co \\ 2. Programa de ingeniería Agroecológica, Universidad de la Amazonia, Florencia - Caquetá, Colombia; \\ juansuarez1@gmail.com \\ * Correspondencia
}

Recibido 16-VII-2020. Corregido 13-X-2020. Aceptado 21-X-2020.

\begin{abstract}
Soil macrofauna and edaphoclimatic conditions in an altitude gradient of coffee growing regions, Huila, Colombia. Introduction: Soil macrofauna plays a key role in soil processes and therefore regulates the supply of ecosystem services; however, its level of activity depends largely of the edaphoclimatic conditions. Objective: This work evaluated the effect of altitude gradient on soil macrofauna communities and edafoclimatic properties in coffee zones of North Huila. Methods: The study included 12 coffee lots separated in two altitude gradients: low (1300-1600 masl) and high (1600-1900 masl). Soil macrofauna was collected by means of monoliths $(25 \times 25 \mathrm{~cm}$ with $30 \mathrm{~cm}$ depth) and edaphoclimatic conditions were studied. Results: A total of 9520 individuals $\mathrm{m}^{-2}$ and a specific richness of 14 taxa were registered. The edaphoclimatic conditions with the greatest statistical difference between altitude gradients were the environmental temperature and relative humidity with differences of $4.9^{\circ} \mathrm{C}$ and $10.4 \%$, respectively. Conclusions: The highest abundance of Coleoptera, Hemiptera, Isoptera, Lepidoptera was presented in warmer sites, that is, altitude gradient 1300-1600 masl, while Chilopoda and Diplopoda adapted better to colder sites in altitude gradient 1600-1900 m.a.s.1.
\end{abstract}

Key words: soil biodiversity; elevation; climate; coffee.

Machado Cuellar, L., Rodríguez Suárez, L., Murcia Torrejano, V., Orduz Tovar, S.A., Ordoñez Espinosa, C.M., \& Suárez, J.C. (2020). Macrofauna del suelo y condiciones edafoclimáticas en un gradiente altitudinal de zonas cafeteras, Huila, Colombia. Revista de Biología Tropical, 69(1), 102-112. DOI 10.15517/rbt.v69i1.42955

La macrofauna del suelo es responsable de una serie de funciones ecológicas y servicios ecosistémicos únicos, que incluye la descomposición de la materia orgánica, el secuestro de carbono orgánico, el ciclaje de nutrientes, el mantenimiento de la estructura del suelo y la infiltración del agua en el suelo (Lavelle et al., 2006; Ruiz, Lavelle, \& Jimenez, 2008; Gholami et al., 2016). Por la importancia de estas funciones vitales para los ecosistemas, la macrofauna del suelo ha recibido mucha atención en medio de un contexto de cambio global (Coyle et al., 2007).

En general, las comunidades de macrofauna del suelo a menudo son consideradas como bioindicadores de la calidad del suelo, por ser sensibles a los cambios ambientales que pueden ocasionar variación en su abundancia y composición (Lavelle et al., 2006; Velasquez \& Lavelle, 2019). A nivel global, el cambio 
climático se ha convertido en un tema principal para estudiar la ecología del suelo, dado que se ha demostrado que las funciones proporcionadas por la macrofauna del suelo dependen del clima (García-Palacios, Maestre, Kattge, \& Wall, 2013; Yin, Qiu, Jiang, \& Wang, 2017). En particular la temperatura, desempeñan un papel importante a lo largo del gradiente altitudinal y afectan la dinámica de la macrofauna del suelo (Tan et al., 2013).

Sumado a esto, el cambio de uso del suelo se cataloga como una de las mayores amenazadas para la biodiversidad del suelo (Nielsen, Wall, \& Six, 2015). De hecho, se ha demostrado que las comunidades de macrofauna son susceptibles al cambio de uso de la tierra (de Graaff, Hornslein, Throop, Kardol, \& Van Diepen, 2019). La preocupación está dirigida hacia sistemas agrícolas en monocultivo donde existe mayor intensificación; por ejemplo, en Colombia los productores de las zonas cafeteras tienen preferencia a sembrar el cultivo en sistemas tecnificados (Escobar, Lagos, Bacca, \& Muñoz, 2016), los cuales requieren una mayor cantidad de productos de síntesis química (fertilizantes y pesticidas) que influyen negativamente en la calidad del suelo (Sauvadet et al., 2019).

En este contexto, se entiende que la composición de la macrofauna del suelo depende de factores climáticos, especies de plantas y propiedades del suelo. Además, estos factores difieren a lo largo de un gradiente de altitud y, por consiguiente, inciden en la estructura, función y composición de la macrofauna del suelo (Tang, 2006; Karungi et al., 2018). Descifrar como varía la abundancia y composición de la macrofauna del suelo en el cultivo de café y en condiciones edafoclimáticas particulares puede ser de utilidad para la implementación de prácticas sostenibles que promuevan entornos favorables para aumentar sus funciones ecológicas.

En este sentido, el objetivo de este estudio fue evaluar el efecto del gradiente de altitud sobre comunidades de macrofauna del suelo y propiedades edafoclimáticas en zonas cafeteras del Norte del Huila. Se planteó que el gradiente altitudinal incide sobre las condiciones climáticas y propiedades del suelo en los cafetales, y a su vez, estos factores inciden en la composición y abundancia de la macrofauna del suelo.

\section{MATERIALES Y MÉTODOS}

Área de estudio: El estudio se realizó en Iquira, Tello y Algeciras, municipios localizados al norte del Huila-Colombia zona que presenta régimen de lluvia bimodal con mayor intensidad en marzo a mayo y octubre a diciembre (IDEAM, 2018). En estos municipios durante agosto de 2018 que corresponde a un periodo seco, se seleccionaron lotes de café arábigo (Coffea arabica) en etapa productiva los cuales estaban sembrados a una densidad de 2222 planta/ha $(2.25 \mathrm{~m}$ entre surcos x 1.5 $\mathrm{m}$ entre planta) y asociados con plátano (Musa paradisiaca L.). Estos lotes de café se ubicaron por encima de los 1300 m.s.n.m, en zonas de ladera con pendientes del 40-75\%.

Diseño de los tratamientos: Se seleccionaron 12 lotes de $10000 \mathrm{~m}^{2}$ cada uno, distribuidos en dos gradientes de altitud bajo (1300-1600 m.s.n.m) y alto (1600-1900 m.s.n.m) para determinar el efecto de la altitud sobre comunidades de macrofauna, variables climáticas y propiedades físicas y químicas del suelo; cada gradiente de altitud contó con un total de seis repeticiones.

Comunidades de macrofauna: La abundancia de los grupos de macrofauna edáfica se evaluó como individuos $\mathrm{m}^{-2}$. Para ello, se recolectaron los individuos usando el método estandarizado ISO 23611-5 (ISO, 2011). En cada lote de café, por cada gradiente de altitud, se tomó tres monolitos (bloques de suelo de 25 x $25 \mathrm{~cm}$ con $30 \mathrm{~cm}$ de profundidad) de manera aleatoria en dirección a la pendiente para cubrir los $10000 \mathrm{~m}^{2}$ de cada lote de café. Cada monolito fue dividido en tres profundidades (0-10, 10-20 y 20-30 cm), y se revisó directamente en el sitio de muestreo, todos los individuos recolectados fueron preservados en alcohol etílico al $96 \%$. La macrofauna del suelo fue agrupada 
según su morfología e identificada a nivel de orden o familia.

Propiedades edafoclimáticas: Las variables climáticas consideradas fueron temperatura ambiente y humedad relativa, las cuales fueron medidas por triplicado utilizando un termohigrometro digital (Serie P 330) en simultáneo al muestreo de la macrofauna del suelo. Se conservaron muestras de suelo compuestas (mezcla de suelo de los tres monolitos usadas para la macrofauna) en cada lote de café para determinar textura del suelo (arena, limo y arcilla) por el método del hidrómetro (Bouyoucos, 1962); pH en agua y la conductividad eléctrica (CE) por potenciometría [1:1]; capacidad de intercambio catiónico (CIC) por titulación con hidróxido de sodio $(\mathrm{NaOH})$ y extracción con acetato de amonio (IGAC, 2006); aluminio (Al) intercambiable por titulación con $\mathrm{NaOH}$ y extracción con $\mathrm{KCl} 1 \mathrm{~N}$; carbono orgánico (CO) por el método Walkley y Black (1934); nitrógeno (N) total por digestión de Kjeldahl (1883) y fósforo $(\mathrm{P})$ disponible por el método Bray-II y espectrofotometría (Bray \& Kurtz, 1945). En campo, al lado de cada monolito de macrofauna, se extrajo un núcleo de suelo inalterado con cilindros de acero inoxidable (altura $5 \mathrm{~cm}$ y ø de $5 \mathrm{~cm}$ ) para determinar la densidad aparente (Blake \& Hartge, 1986) y humedad del suelo (IGAC, 2006).

Análisis de datos: La abundancia de la macrofauna edáfica se evaluó mediante modelos lineales generalizados (GLM) con una función de regresión negativa binomial (Sileshi, 2008) debido a la presencia de valores ceros que no permiten un ajuste con distribución normal. Los datos de variables edafoclimáticas fueron analizados utilizando análisis de varianza mediante una prueba LSD de Fisher considerando como factor el gradiente altitudinal. A partir de las matrices de datos de comunidades de macrofauna y propiedades edafoclimáticas se realizó el análisis de componentes principales (ACP) para explorar las tendencias de relacionamiento de las variables en función del gradiente altitudinal. Por último, se calculó las relaciones de las diferentes variables utilizando correlación de Pearson. Los análisis estadísticos se desarrollaron usando los paquetes nlme, ggplot2 y ade4 del lenguaje de programación R 3.6.1 (R Core Team, 2019) y la interfaz de InfoStat (Di Rienzo et al., 2018).

\section{RESULTADOS}

Comunidad de macrofauna: En total se registraron $9520 \mathrm{ind} / \mathrm{m}^{2}$, con rangos entre 32 y $736 \mathrm{ind} / \mathrm{m}^{2}$ por muestra de suelo. La riqueza total fue de 14 táxones y se destaca los grupos Formicidae $(34.1 \%)$ y Oligochaeta $(31.1 \%)$ por representar el $65.2 \%$ de la macrofauna recolectada (Fig. 1).

$\mathrm{Al}$ analizar el efecto del gradiente altitudinal se encontraron diferencias significativas $(\mathrm{P}<0.05)$ para seis táxones (Tabla 1). De acuerdo con su abundancia media los táxones Coleoptera, Hemiptera, Isoptera, Lepidoptera y la categoría "otros" predominaron en el gradiente de altitud 1300-1600 m.s.n.m, mientras que el taxon Chilopoda fue más abundante en el gradiente de altitud 1600-1900 m.s.n.m. Además, Diplopoda estuvo presente solamente en el gradiente 1600-1900 m.s.n.m (Tabla 1).

El análisis de componentes principales para la comunidad de macrofauna del suelo explicó el $47.4 \%$ de la variabilidad de los datos con los dos primeros ejes. En el eje 1, la proyección de los vectores de Lepidoptera y Isopoda se relacionaron con el gradiente altitudinal 1300-1600 m.s.n.m (Fig. 2). En el eje 2, la proyección de los vectores Oligochaeta y Chilopoda indicaron su relación con el gradiente altitudinal 1600-1900 m.s.n.m, mientras que el vector de Formicidae e Isoptera revelaron relación con el gradiente altitudinal 1300-1600 m.s.n.m (Fig. 2). Al determinar la separación del gradiente altitudinal en función de los grupos de macrofauna no se encontró un efecto significativo según la prueba de Monte Carlo $(\mathrm{P}=0.89)$.

Propiedades edafoclimáticas: El gradiente de altitud influyó significativamente sobre las variables climáticas $(\mathrm{P}<0.05)$. La 


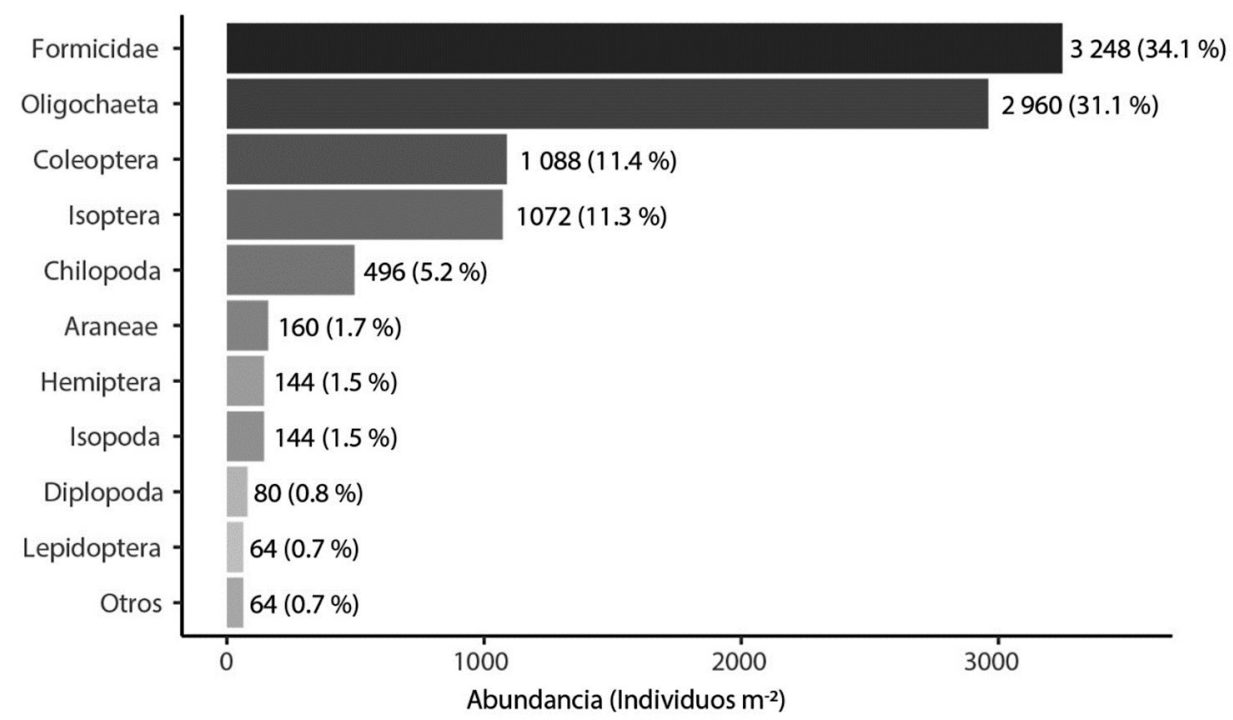

Fig. 1. Abundancia total (ind $/ \mathrm{m}^{2}$ ) y frecuencia relativa (\%) de la comunidad de macrofauna del suelo de cafetales cultivados en zonas de ladera. La categoría "otros" hace referencia a individuos con una frecuencia muy baja $(<5 \%)$ como Blattodea, Dermaptera, Gastropoda y Orthoptera.

Fig. 1. Total abundance (ind $/ \mathrm{m}^{2}$ ) and relative frequence (\%) of soil macrofauna community from hillside cultivated coffee fields. "Otros" category are groups with low frequency $(<5 \%)$, like Blattodea, Dermaptera, Gastropoda and Orthoptera.

TABLA 1

Abundancia de la macrofauna del suelo en cafetales en dos gradientes altitudinales en el Norte del Huila (Media \pm EE)

TABLE 1

Coffee field macrofauna abundance on two altitudinal gradients in the North of Huila (Mean \pm SE)

\begin{tabular}{lccc}
\multicolumn{1}{c}{ Grupos de macrofauna } & 1300 -1 600 m.s.n.m & $1600-1900$ m.s.n.m & p-valor \\
Araneae & $3.81 \pm 0.4$ & $4.4 \pm 0.5$ & 0.335 \\
Coleoptera & $29.71 \pm 1.1$ & $25.78 \pm 1.2$ & $\mathbf{0 . 0 2 6}$ \\
Chilopoda & $6.86 \pm 0.5$ & $19.56 \pm 1$ & $\mathbf{0 . 0 0 1}$ \\
Diplopoda & & $4.44 \pm 0.5$ & 0.819 \\
Formicidae & $87.63 \pm 29.3$ & $78.22 \pm 28.2$ & $\mathbf{0 . 0 0 4}$ \\
Hemiptera & $4.57 \pm 0.4$ & $2.67 \pm 0.3$ & 0.683 \\
Isopoda & $3.81 \pm 0.4$ & $3.56 \pm 0.4$ & $\mathbf{0 . 0 0 1}$ \\
Isoptera & $34.29 \pm 1.2$ & $19.56 \pm 1.0$ & $\mathbf{0 . 0 0 2}$ \\
Lepidoptera & $2.29 \pm 0.3$ & $0.89 \pm 0.2$ & 0.840 \\
Oligochaeta & $72.38 \pm 24.2$ & $80 \pm 29$ & $\mathbf{0 . 0 0 2}$ \\
Otros & $2.29 \pm 0.3$ & $0.89 \pm 0.2$ & 0.842 \\
Abundancia total & $245.33 \pm 36.9$ & $234.67 \pm 38.2$ & 0.581 \\
Riqueza & $3.33 \pm 0.4$ & $3.67 \pm 0.4$ & \\
\hline
\end{tabular}

temperatura ambiente en el gradiente de altitud 1300-1600 m.s.n.m obtuvo un promedio mayor de $23.79^{\circ} \mathrm{C}$ y una diferencia de $4.98^{\circ} \mathrm{C}$ en referencia al valor encontrado en el gradiente altitudinal alto $\left(18.81{ }^{\circ} \mathrm{C}\right.$, Tabla 2$)$. En cuanto, la humedad relativa en el gradiente de altitud 1600-1900 m.s.n.m presento un promedio mayor de $79.45 \%$, y una diferencia de $10.4 \%$ respecto al gradiente de altitud bajo (69.05\%, Tabla 2). La capacidad de 


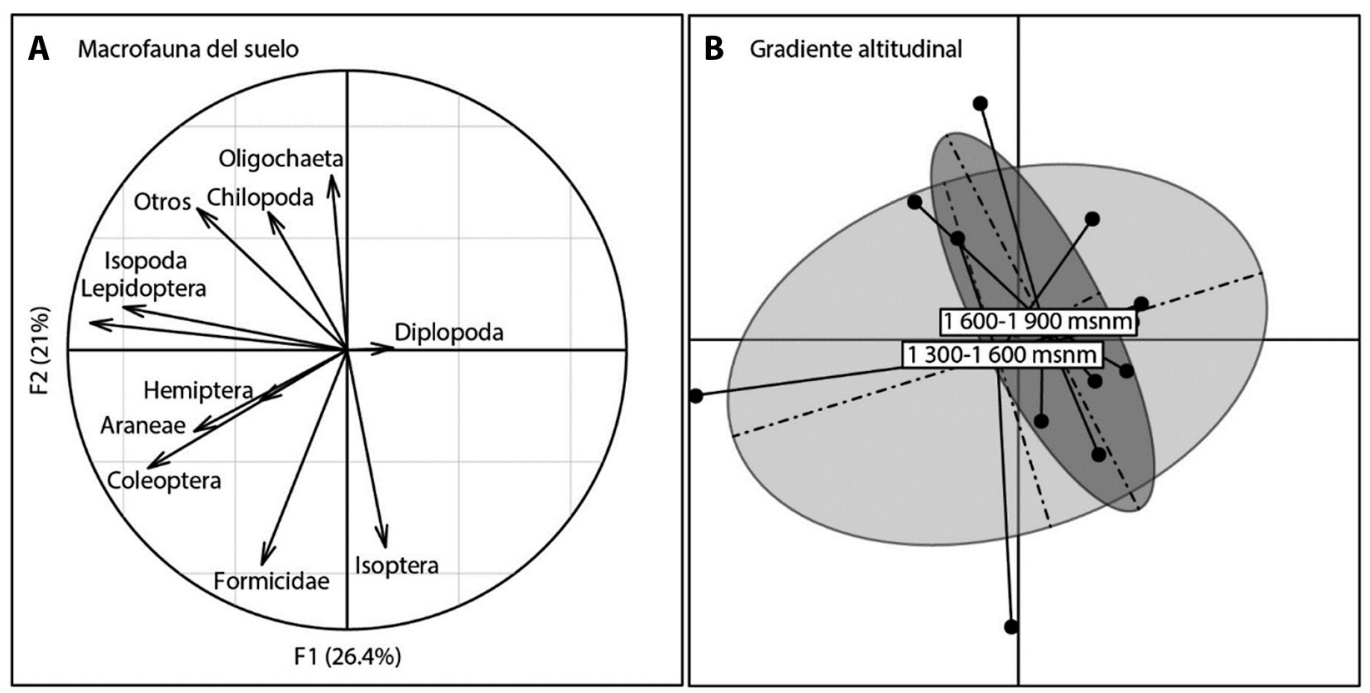

Fig. 2. A. Proyección en el plano factorial F1/F2 de las variables macrofauna del suelo. B. Proyección en el plano factoria F1/F2 de los puntos de muestreo agrupados según el gradiente de altitud.

Fig. 2. A. Projection in the factorial plane F1/F2 of the soil macrofauna variables. B. Projection in the factorial plane F1/F2 of the sampling points grouped by the altitude.

TABLA 2

Condiciones edafoclimáticas en cafetales en dos gradientes altitudinales en el Norte del Huila (Media \pm EE)

TABLE 2

Edaphoclimatic conditions on coffee fields on two altitudinal gradients in the North of Huila (Mean \pm SE)

\begin{tabular}{|c|c|c|c|c|}
\hline & & 1300-1600 m.s.n.m & 1600-1900 m.s.n.m & $\mathrm{P}$ \\
\hline \multirow[t]{2}{*}{ Variables climáticas } & Temperatura $\left({ }^{\circ} \mathrm{C}\right)$ & $23.79 \pm 0.4$ & $18.81 \pm 0.5$ & 0.001 \\
\hline & Humedad relativa $(\%)$ & $69.05 \pm 2.4$ & $79.45 \pm 2.6$ & 0.016 \\
\hline \multirow[t]{12}{*}{ Propiedades del suelo } & Humedad suelo (\%) & $43.65 \pm 4$ & $49.98 \pm 4.3$ & 0.306 \\
\hline & $\mathrm{Da}\left(\mathrm{g} \mathrm{cm}^{-3}\right)$ & $1.35 \pm 0.05$ & $1.41 \pm 0.05$ & 0.388 \\
\hline & Arena (\%) & $60.42 \pm 3.5$ & $70.62 \pm 3.5$ & 0.059 \\
\hline & Limo (\%) & $24.47 \pm 2.6$ & $16.53 \pm 2.8$ & 0.062 \\
\hline & Arcilla (\%) & $15.11 \pm 1.5$ & $12.84 \pm 1.6$ & 0.348 \\
\hline & $\mathrm{pH}$ & $5.43 \pm 0.2$ & $5.65 \pm 0.2$ & 0.523 \\
\hline & $\mathrm{CE}\left(\mathrm{dS} \mathrm{m} \mathrm{m}^{-1}\right)$ & $0.37 \pm 0.05$ & $0.27 \pm 0.06$ & 0.202 \\
\hline & $\mathrm{CIC}$ & $29.28 \pm 2.1$ & $20.69 \pm 2.2$ & 0.018 \\
\hline & $\mathrm{Al}\left(\mathrm{cmol}+\mathrm{kg}^{-1}\right)$ & $0.33 \pm 0.09$ & $0.33 \pm 0.1$ & 0.99 \\
\hline & $\mathrm{CO}(\%)$ & $2.95 \pm 0.4$ & $3.10 \pm 0.4$ & 0.809 \\
\hline & $\mathrm{N}$ total $(\%)$ & $0.25 \pm 0.04$ & $0.25 \pm 0.04$ & 0.986 \\
\hline & $\mathrm{P}\left(\mathrm{mg} \mathrm{kg}^{-1}\right)$ & $3.99 \pm 1.7$ & $10.6 \pm 1.8$ & 0.022 \\
\hline
\end{tabular}

Da: densidad aparente, CE: conductividad eléctrica, CIC: capacidad de intercambio catiónico.

intercambio catiónico (CIC) y el fósforo disponible fueron las únicas propiedades químicas que presentaron diferencias significativas con respecto al gradiente de altitud. La CIC fue significativamente mayor en el rango de altitud 1300-1600 m.s.n.m, y el fósforo significativamente mayor en el gradiente de altitud 1600-1900 m.s.n.m (Tabla 2).

El análisis de componentes principales para las propiedades edafoclimáticas explicó el 
$59.3 \%$ de la variabilidad de los datos con los dos primeros ejes. El eje 1 proyectó los vectores de humedad relativa, fósforo y arena que estuvieron asociados con el gradiente de altitud 1600-1900 m.s.n.m, mientras que los vectores de temperatura, CIC y limo se relacionaron con el gradiente altitudinal 1300-1600 m.s.n.m (Fig. 3); aunque no se encontraron diferencias estadísticas en propiedades intrínsecas del suelo como la textura particularmente arena y limo, su valor promedio aportó en cierta manera la separación de los tratamientos en eje 1. El eje 2 proyectó los vectores de $\mathrm{CO}$ y $\mathrm{N}$ con una asociación débil con el gradiente 1300-1600 m.s.n.m (Fig. 3). La separación de los cafetales entre los rangos de altitud a partir de los variables climáticas y propiedades del suelo fue significativa según la prueba de Monte Carlo (p-valor: 0.007).

Relación de la macrofauna con las propiedades edafoclimáticas: Los coeficientes de correlación revelaron asociaciones significativas entre los grupos de macrofauna y las condiciones edafoclimáticas estudiadas (Fig. 4).
La abundancia de Coleoptera se correlacionó negativamente con la textura del suelo arena $(\mathrm{R}$ $\left.=-0.58^{*}\right)$; Chilopoda positivamente con humedad relativa $\left(\mathrm{R}=0.6^{*}\right)$ y fósforo $\left(\mathrm{R}=0.7^{*}\right)$, pero negativamente con la temperatura $(\mathrm{R}=$ $\left.-0.56^{*}\right)$; Diplopoda negativamente con CIC (R $\left.=-0.57^{*}\right)$; Isopoda positivamente con fósforo $\left(\mathrm{R}=0.60^{*}\right)$ y Oligochaeta negativamente con $\mathrm{pH}\left(\mathrm{R}=-0.57^{*}\right)$.

\section{DISCUSIÓN}

Comunidades de macrofauna: La mayor abundancia presente de hormigas (Formicidae) y lombrices (Oligochaeta) en los cafetales independiente del gradiente altitudinal, indica su amplia distribución (Santos et al., 2018, Rodriguez, Cuaran, \& Suarez, 2019). Estos organismos considerados ingenieros del suelo (lombrices, hormigas y termitas) mejoran la estructura del suelo (Lavelle et al., 2006), además desempeñan una importante función en la dinámica de los nutrientes al contribuir en la aireación, drenaje, descomposición, aporte de carbono y depredación de insectos, como
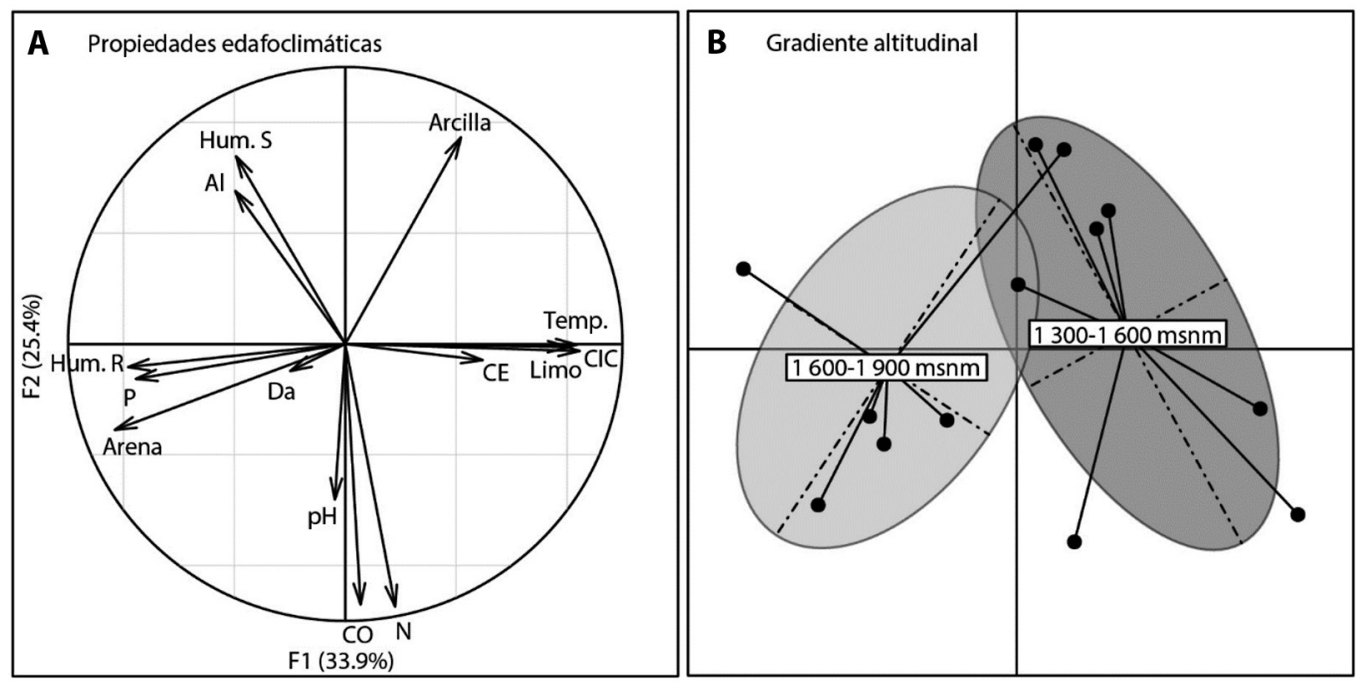

Fig. 3. A. Proyección en el plano factorial F1/F2 de las propiedades edafoclimáticas. B. Proyección en el plano factorial F1/ F2 de los puntos de muestreo agrupados según el gradiente altitudinal. Temp.: Temperatura, Hum. R: Humedad relativa y Hum. S: Humedad del suelo.

Fig. 3. A. Projection in the factorial plane F1/F2 of the edaphoclimatic properties. B. Projection in the factorial plane F1/F2 of the sampling points grouped by the altitude. Temp.: Temperature, Hum. R: Relative humidity y Hum. S: Soil humidity. 


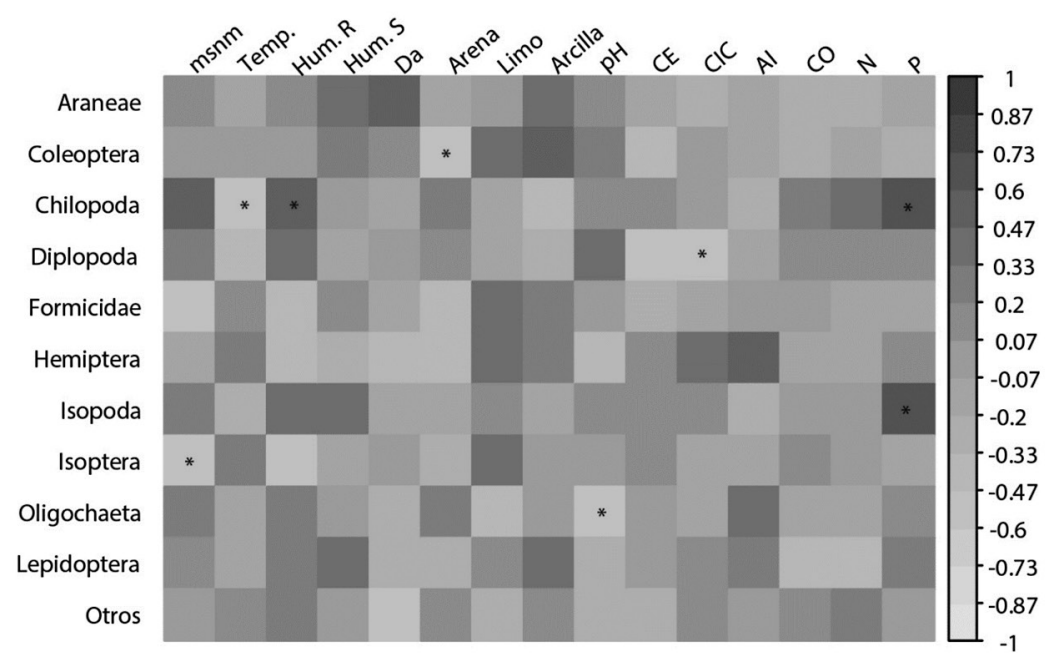

Fig. 4. Mapa de calor de los coeficientes de correlación entre macrofauna del suelo y propiedades edafoclimáticas. La escala de grises representa la dirección y la fuerza de la correlación, * indica correlación significativa al $5 \%$ de probabilidad. Temp: temperatura ambiente, Hum R: humedad relativa y Hum S: humedad del suelo.

Fig. 4. Heatmap of correlation coefficients between soil macrofauna and edaphoclimatic properties. The grey scale represents the direction and strength of the correlation, * indicates significative correlation at $5 \%$ of probability. Temp.: Temperature, Hum. R: Relative humidity y Hum. S: Soil humidity.

prestación de servicios a los agroecosistemas de café (Gathorne, Syaukani, \& Eggleton, 2001; Karungi et al., 2018).

Los ciempiés (Chilopoda) que son depredadores y reguladores de poblaciones presentaron mejor adaptación en el gradiente de altitud alto. Al respecto, de Oliveira et al. (2019) menciona que los miriápodos (Chilopoda y Diplopoda) se benefician en condiciones de mayor humedad y temperaturas más estables, lo que se confirma para la altitud 1600-1900 m.s.n.m. Incluso, los milpiés (Diplopoda) que juegan un papel importante en la dinámica de la materia orgánica por medio de la fragmentación de la hojarasca, solo estuvieron presente en la altitud 1600-1900 m.s.n.m. Al examinar la respuesta de Diplopoda a condiciones de humedad del suelo Collison, Riutta, y Slade (2013) concluyeron que su actividad disminuye a bajos niveles de humedad, lo cual pudiera explicar la ausencia de este grupo en la altitud baja.

Los chinches (Hemiptera) al ser fitófagos pueden generar efectos negativos en el rendimiento y calidad del grano de café (Ahmed,
Murungi, \& Babin, 2016). Su mayor abundancia en el gradiente 1300-1600 m.s.n.m se encuentra asociado a las condiciones climáticas, ya que según Bigirimana, Uzayisenga, y Gut (2019) en regiones cafeteras el aumento de la temperatura promueve significativamente la abundancia de los hemípteros especialmente de la familia Pentatomidae, incluso se ha informado que las mejores tasas de crecimiento y reproducción de este grupo se obtienen dentro de un rango de temperatura de $20-25{ }^{\circ} \mathrm{C}$ (Ahmed et al., 2016). Lo cual es consistente con la temperatura registrada en este gradiente altitudinal de $23.7^{\circ} \mathrm{C}$.

Las termitas (Isoptera) son muy susceptibles a las condiciones climáticas. De hecho, la temperatura ambiente es un predictor fuerte de la actividad de las termitas (Cancello, Silva, Vasconcellos, Reis, \& Oliveira, 2014). En los cafetales, su mayor abundancia se presentó en el gradiente altitudinal más bajo (1300-1600 m.s.n.m). Al respecto, Gathorne et al. (2001) informan que la altitud es una variable que tiene un efecto significativo negativo sobre la 
diversidad y abundancia de especies de termitas, la cual puede disminuir con el aumento de 100 m.s.n.m.

Los grupos asociados a la hojarasca como Lepidoptera y otros (Blattodea, Dermaptera, Gastropoda y Orthoptera) presentaron su mayor abundancia en cafetales con gradiente de altitud más bajo. Lo que concuerda con Salinas et al. (2011) quienes afirman que en condiciones cálidas con mayor temperatura las tasas de descomposición de la hojarasca se aceleran (Salinas et al., 2011), indicativo de una mayor actividad de grupos funcionales de la macrofauna de tipo detritívoro depredador (Illig, Schatz, Scheu, \& Maraun, 2008).

Propiedades edafoclimáticas: La disminución de la temperatura ambiente y el aumento de la humedad relativa con relación al gradiente altitudinal confirmó las tendencias descritas acerca de la incidencia de la altitud sobre el microclima (Hodkinson, 2005). Particularmente, se encontró en los cafetales diferencias de $4.9{ }^{\circ} \mathrm{C}$ y $10.4 \%$ de humedad respecto a los gradientes bajo (1300-1600 m.s.n.m) y alto (1600-1 900 m.s.n.m). Resultados similares fueron publicados para cafetales por Karungi et al. (2018) donde mencionan una diferencia de temperatura de $2.2^{\circ} \mathrm{C}$, entre gradientes bajo (1300-1499 m.s.n.m) y alto (1 680-2 100 m.s.n.m).

La capacidad de intercambio catiónico (CIC) fue mayor en el gradiente altitudinal bajo, lo cual puede estar asociado a una mayor proporción de arcillas en estos sitios, teniendo en cuenta que estas partículas minerales poseen gran área superficial y cargas eléctricas que confieren la capacidad del suelo para retener cationes como $\mathrm{Ca}^{2+}, \mathrm{Mg}^{2+}, \mathrm{Na}^{+}$y K $\mathrm{K}^{+}$(Khaledian et al., 2017). Por otra parte, se resalta un mayor contenido de fósforo disponible en el gradiente altitudinal alto (1600-1900 m.s.n.m), siendo similar a lo publicado por Szopka, Kabala, Karczewska, Bogacz, y Jeziersk, (2010) quienes indican un aumento significativo del fósforo disponible a la altitud mayor. Asimismo, autores como Mou et al. (2020) asocian estos resultados con la disminución de la temperatura ambiente a lo largo del gradiente y la precipitación que tienen incidencia directa en la meteorización de los minerales que contiene el fosforo del suelo.

Relación entre macrofauna del suelo y propiedades edafoclimáticas: Se encontró que la textura gruesa (arenas) afectó negativamente la densidad de coleópteros. Lo que concuerda con Trujillo, Carrillo, Rivas, y Hernández (2016) quienes informan mayor supervivencia de larvas de Coleoptera en suelo de textura arcillosa, debido a que la textura fina favorece las actividades excavación y anidación.

La relación positiva encontrada entre los Chilopoda e Isopoda con el fósforo disponible confirma que la macrofauna del suelo regula en gran medida la dinámica de los nutrientes mediante sus grupos funcionales como detritívoros y depredadores (Velasquez \& Lavelle, 2019). Sin embargo, el papel de las lombrices y termitas en el ciclo del fósforo ha sido estudiado con mayor frecuencia. Según ChapuisLardy, Le Bayon, Brossard, López-Hernández y Blanchart (2011) estos organismos producen estructuras biogénicas, como moldes de lombrices y montículos de termitas, en los que los contenidos y las formas de $\mathrm{P}$ difieren del suelo circundante.

Se considera que la relación negativa entre lombrices y pH del suelo reflejó la adaptación de este grupo a suelos ácidos, que para los cafetales estudiados se clasifica como fuertemente acido. Precisamente, el pH es considerado un predictor importante de las poblaciones de lombrices (Umiker, Johnson-Maynard, Hatten, Eigenbrode, \& Bosque-Pérez, 2009). En estudios realizados por McCallum et al. (2016) encontraron que la abundancia de lombrices disminuyó en $\mathrm{pH}<4.5$ en combinación con bajos contenidos de materia orgánica.

Como conclusión de este estudio se encontró que los cambios en la abundancia de macrofauna del suelo en los cafetales están asociados en gran parte a condiciones climáticas, basado en las amplias diferencias encontradas para temperatura ambiente y humedad relativa en los gradientes de altitud. Así mismo, los 
coeficientes de correlación de la macrofauna con propiedades edafoclimáticas confirmaron la dependencia de la macrofauna del suelo a factores ambientales y su potencial influencia en el ciclaje de nutrientes mediante sus roles funcionales.

\section{AGRADECIMIENTOS}

Al Servicio Nacional de Aprendizaje SENA, Sistema de Investigación, Desarrollo Tecnológico e Innovación - SENNOVA, Centro de Formación Agroindustrial - Regional Huila y en especial a los productores de los municipios de Iquira, Tello y Algeciras, que permitieron el desarrollo de la investigación en cada uno de sus lotes de café.

\section{RESUMEN}

Introducción: La macrofauna del suelo cumple un rol clave en los procesos del suelo, por lo tanto, regula la oferta se servicios ecosistémicos; sin embargo, su nivel de actividad depende en gran parte de las condiciones edafoclimáticas. Objetivo: El presente trabajo evaluó el efecto del gradiente de altitud sobre comunidades de macrofauna del suelo y propiedades edafoclimáticas en zonas cafeteras del Norte del Huila. Métodos: El estudio incluyó 12 lotes de café separados en dos gradientes de altitud: bajo (1300-1600 m.s.n.m) y alto (1600-1900 m.s.n.m), se recolectó la macrofauna del suelo mediante monolitos (25 x $25 \mathrm{~cm}$ a $30 \mathrm{~cm}$ de profundidad) y se estudiaron las condiciones edafoclimáticas. Resultados: En total se registró 9520 individuos $\mathrm{m}^{-2} \mathrm{y}$ una riqueza específica de 14 táxones. Las condiciones edafoclimáticas con mayor diferencia estadística entre los gradientes de altitud, fue la temperatura ambiente y humedad relativa con diferencias de $4.9{ }^{\circ} \mathrm{C}$ y $10.4 \%$, respectivamente. Conclusiones: La mayor abundancia de Coleoptera, Hemiptera, Isoptera y Lepidoptera se presentó en sitios más cálidos, es decir a un gradiente de altitud entre 1300-1600 m.s.n.m, mientras que Chilopoda y Diplopoda se adaptaron mejor a sitios más fríos en el gradiente altitudinal entre 1600-1900 m.s.n.m.

Palabras clave: biodiversidad edáfica; elevación; clima; café.

\section{REFERENCIAS}

Ahmed, A.G., Murungi, L.K., \& Babin, R. (2016). Developmental biology and demographic parameters of antestia bug Antestiopsis thunbergii (Hemiptera: Pentatomidae), on Coffea arabica (Rubiaceae) at different constant temperatures. International Journal of Tropical Insect Science, 36(3), 119-127.

Bigirimana, J., Uzayisenga, B., \& Gut, L.J. (2019). Population distribution and density of Antestiopsis thunbergii (Hemiptera: Pentatomidae) in the coffee growing regions of Rwanda in relation to climatic variables. Crop Protection, 122, 136-141.

Bray, R.H., \& Kurtz, L.T. (1945). Determination of total, organic, and available forms of phosphorus in soils. Soil science, 59(1), 39-46.

Blake, G.R., \& Hartge, K.H. (1986). Bulk density. In A. Klute (Ed.), Methods of Soil Analysis-Physical and Mineralogical Methods (pp. 493-544.). EE. UU: Wiley \& Sons.

Bouyoucos, G.I. (1962). Hydrometer method improved for making particle size analysis of soils. Agronomy Journal, 54(3), 464-465.

Cancello, E.M., Silva, R.R., Vasconcellos, A., Reis, Y.T., \& Oliveira, L.M. (2014). Latitudinal variation in termite species richness and abundance along the Brazilian Atlantic Forest hotspot. Biotropica, 46(4), 441-450.

Chapuis-Lardy, L., Le Bayon, R.C., Brossard, M., LópezHernández, D., \& Blanchart, E. (2011). Role of soil macrofauna in phosphorus cycling. In E. Bünemann, A. Oberson, \& E. Frossard (Eds.), Phosphorus in Action (pp. 199-213). Berlin: Springer.

Collison, E.J., Riutta, T., \& Slade, E.M. (2013). Macrofauna assemblage composition and soil moisture interact to affect soil ecosystem functions. Acta Oecologica, 47, 30-36.

Coyle, D.R., Nagendra, U.J., Taylor, M.K., Campbell, J.H., Cunard, C.E., Joslin, A.H., \& Callaham Jr, M.A. (2017). Soil fauna responses to natural disturbances, invasive species, and global climate change: Current state of the science and a call to action. Soil Biology and Biochemistry, 110, 116-133.

de Graaff, M.A., Hornslein, N., Throop, H.L., Kardol, P., \& Van Diepen, L.T. (2019). Effects of agricultural intensification on soil biodiversity and implications for ecosystem functioning: a meta-analysis. Advances in Agronomy, 155, 1-44.

De Oliveira, M.P.A., Bastos-Pereira, R., Torres, S.H.S., Pereira, T.S., Batista, F.M., Alves, J.P., \& Pietrobon, T. (2019). Choosing sampling methods for Chilopoda, Diplopoda and Isopoda (Oniscidea): A case study for ferruginous landscapes in Brazilian Amazonia. Applied Soil Ecology, 143, 181-191.

Di Rienzo, J.A., Casanoves, F., Balzarini, M.G., Gonzalez, L., Tablada, M., \& Robledo, C.W. (2018). InfoStat. Centro de Transferencia InfoStat, FCA, Universidad Nacional de Córdoba, Argentina. Recuperado de http://www.infostat.com.ar 
Escobar, H., Lagos, T.C., Bacca, T., \& Muñoz, J.A. (2016). Caracterización de los sistemas productivos de café en Nariño, Colombia. Revista UDCA Actualidad \& Divulgación Científica, 19(1), 105-113.

García-Palacios, P., Maestre, F.T., Kattge, J., \& Wall, D.H. (2013). Climate and litter quality differently modulate the effects of soil fauna on litter decomposition across biomes. Ecology Letters, 16(8), 1045-1053.

Gathorne, F.H., Syaukani, \& Eggleton P. (2001). The effects of altitude and rainfall on the composition of the termites (Isoptera) of the Leuser Ecosystem (Sumatra, Indonesia). Journal of Tropical Ecology, 17(3), 379-393.

Gholami, S., Sayad, E., Gebbers, R., Schirrmann, M., Joschko, M., \& Timmer, J. (2016). Spatial analysis of riparian forest soil macrofauna and its relation to abiotic soil properties. Pedobiologia, 59(1-2), 27-36.

Hodkinson, I.D. (2005). Terrestrial insects along elevation gradients: species and community responses to altitude. Biological reviews, 80(3), 489-513.

Illig, J., Schatz, H., Scheu, S., \& Maraun, M. (2008). Decomposition and colonization by micro-arthropods of two litter types in a tropical montane rain forest in southern Ecuador. Journal of Tropical Ecology, 24, 157-167.

IGAC. (2006). Métodos analiticos del laboratorio de suelos (Sexta edición). Bogotá, Colombia: Instituto Geográfico Agustín Codazzi.

IDEAM. (2018). MAPAS: Atlas climatológico de Colombia Colombia: Instituto de Hidrología, Meteorología y Estudios Ambientales. Recuperado de http://www. ideam.gov.co

ISO. (2011). ISO 23611-5. Soil Quality-Sampling of Soil Invertebrates-Part 5. Sampling and Extraction of Soil Macro-invertebrates. Genova, Suecia: International Organization for Standardization.

Karungi, J., Cherukut, S., Ijala, A.R., Tumuhairwe, J.B., Bonabana-Wabbi, J., Nuppenau, E.A., \& Otte, A. (2018). Elevation and cropping system as drivers of microclimate and abundance of soil macrofauna in coffee farmlands in mountainous ecologies. Applied Soil Ecology, 132, 126-134.

Khaledian, Y., Brevik, E.C., Pereira, P., Cerdà, A., Fattah, M.A., \& Tazikeh, H. (2017). Modeling soil cation exchange capacity in multiple countries. Catena, 158, 194-200.

Kjeldahl, J. (1883). A New Method for the Determination of Nitrogen in Organic Matter. Zeitschrift für Analytische Chemie, 22, 366-382.
Lavelle, P., Decaëns, T., Aubert, M., Barot, S., Blouin, M., Bureau, F., Margerie, P., Mora, P., Rossi, J.P. (2006). Soil invertebrates and ecosystem services. European Journal of Soil Biology, 42, S3-S15.

McCallum, H.M., Wilson, J.D., Beaumont, D., Sheldon, R., O’Brien, M.G., \& Park, K.J. (2016). A role for liming as a conservation intervention? Earthworm abundance is associated with higher soil $\mathrm{pH}$ and foraging activity of a threatened shorebird in upland grasslands. Agriculture, Ecosystems \& Environment, 223, 182-189.

Mou, X.M., Wu, Y., Niu, Z., Jia, B., Guan, Z.H., Chen, J., \& Li, X.G. (2020). Soil phosphorus accumulation changes with decreasing temperature along a 2300 $\mathrm{m}$ altitude gradient. Agriculture, Ecosystems \& Environment, 301, 107050.

Nielsen, U.N., Wall, D.H., \& Six, J. (2015). Soil biodiversity and the environment. Annual Review of Environment and Resources, 40, 63-90.

R Core Team (2019). R A language and environment for statistical computing. R Foundation for Statistical Computing, Vienna, Austria. Recuperado de https:// www.R-project.org/.

Rodriguez, L.S., Cuaran, S.P., \& Suarez, J.C.S. (2019). Soil Macrofauna and Edaphic Properties in Coffee Production Systems in Southern Colombia. Floresta e Ambiente, 26(3), 1-8.

Ruiz, N., Lavelle, P., \& Jiménez, J. (2008). Soil macrofauna field manual: technical level. Roma, IT: Food and Agriculture Organization of the United Nations.

Salinas, N., Malhi, Y., Meir, P., Silman, M., Roman Cuesta, R., Huaman, J., \& Farfan, F. (2011). The sensitivity of tropical leaf litter decomposition to temperature: results from a large-scale leaf translocation experiment along an elevation gradient in Peruvian forests. New phytologist, 189(4), 967-977.

Santos, J.B.D., Ramos, A.C., Azevedo Júnior, R., Oliveira Filho, L.C.I.D., Baretta, D., \& Cardoso, E.J.B.N. (2018). Soil macrofauna in organic and conventional coffee plantations in Brazil. Biota Neotropica, 18(2), e20180515.

Sauvadet, M., Van den Meersche, K., Allinne, C., Gay, F., de Melo Virginio Filho, E., Chauvat, M., \& Harmand, J.M. (2019). Shade trees have higher impact on soil nutrient availability and food web in organic than conventional coffee agroforestry. Science of the Total Environment, 649, 1065-1074.

Sileshi, G. (2008). The excess-zero problem in soil animal count data and choice of appropriate models for statistical inference. Pedobiologia, 52(1), 1-17. 
Szopka, K., Kabala, C., Karczewska, A., Bogacz, A., \& Jezierski, P. (2010). Pools of available nutrients in soils from different altitudinal forest zones located in a monitoring system of the Karkonosze Mountains National Park, Poland. Polish Journal of Soil Science, 43(2), 173-188.

Tang, C.Q. (2006). Forest vegetation as related to climate and soil conditions at varying altitudes on a humid subtropical mountain, Mount Emei, Sichuan, China. Ecological Research, 21(2), 174-180.

Tan, B., Wu, F., Yang, W., Yu, S., Liu, L., Wang, A., \& Yang, Y. (2013). Seasonal dynamics of soil fauna in the subalpine and alpine forests of west Sichuan at different altitudes. Acta Ecologica Sinica, 33(1), $12-22$.

Trujillo, M.A., Carrillo, R.H., Rivas, A.S., \& Hernández, R.A. (2016). Structure and composition of the community of beetles (Coleoptera: Scarabaeoidea) from Chacateca's Hill, Zapotitlan, Puebla, Mexico. Revista Mexicana de Biodiversidad, 87(1), 109-122.
Umiker, K.J., Johnson-Maynard, J.L., Hatten, T.D., Eigenbrode, S.D., \& Bosque-Pérez, N.A. (2009). Soil carbon, nitrogen, $\mathrm{pH}$, and earthworm density as influenced by cropping practices in the Inland Pacific Northwest. Soil and Tillage Research, 105(2), 184-191.

Velasquez, E., \& Lavelle, P. (2019). Soil macrofauna as an indicator for evaluating soil based ecosystem services in agricultural landscapes. Acta Oecologica, 100, 103446 .

Walkley, A., \& Black, I.A. (1934). An examination of the Degtjareff method for determining soil organic matter, and a proposed modification of the chromic acid titration method. Soil Science, 37(1), 29-38.

Yin, X., Qiu, L., Jiang, Y., \& Wang, Y. (2017). Diversity and spatial-temporal distribution of soil macrofauna communities along elevation in the Changbai Mountain, China. Environmental Entomology, 46(3), 454-459. 